\title{
The effect of resting transmembrane voltage on cell electropermeabilization: a numerical analysis
}

\author{
Blaž Valič, Mojca Pavlin, Damijan Miklavčič * \\ Faculty of Electrical Engineering, University of Ljubljana, Tržaška 25, SI-1000 Ljubljana, Slovenia
}

Received 22 September 2003; received in revised form 9 December 2003; accepted 11 December 2003

\begin{abstract}
The transmembrane voltage induced due to applied electric field superimposes to the resting transmembrane voltage of the cell. On the part of the cell membrane, where the transmembrane voltage exceeds the threshold transmembrane voltage, changes in the membrane occur, leading to increase in membrane permeability known as electropermeabilization. This part of the cell membrane represents the permeabilized area through which the transport of molecules occurs. In this paper we calculated numerically the permeabilized area for different electric field strength, resting transmembrane voltage, cell shape and cell orientation with respect to the applied electric field.

Results show that when the transmembrane voltage is near the threshold transmembrane voltage, the permeabilized area of the cell is increased on the anodic side and decreased on the cathodic side due to the resting transmembrane voltage. In some cases, only anodic side of the cell is permeabilized. Therefore, by using bipolar pulses, the permeabilized area can be significantly increased and consequentially also the efficiency of electropermeabilization. However, when the induced transmembrane voltage is far above the threshold, the effect of the resting transmembrane voltage is negligible. These observations are valid for different cell shapes and orientations.
\end{abstract}

(C) 2004 Elsevier B.V. All rights reserved.

Keywords: Finite-elements model; Electropermeabilization; Resting transmembrane voltage

\section{Introduction}

One of commonly used techniques for achieving transmembrane transport of molecules which otherwise cannot cross membrane is electropermeabilization, a method where the applied electric field is used to permeabilize cell membrane [1-6]. Under normal physiological conditions and when no electric field is applied, only resting transmembrane voltage exists. When the electric field is applied, the induced transmembrane voltage is superimposed to the resting transmembrane voltage $[2,7,8]$, thus, the resulting transmembrane voltage is a sum of both the resting and the induced transmembrane voltage. When the threshold value of the transmembrane voltage is exceeded, biochemical and physiological changes occur in the cell membrane, which consequently lead to the increased permeability for ions and molecules $[5,9]$. Further increase in applied electric field intensity may cause irreversible membrane permeabilization and cell death.

\footnotetext{
* Corresponding author. Tel.: +386-1-4768456; fax: +386-1-4264-658

E-mail address: damijan@svarun.fe.uni-lj.si (D. Miklavčič).
}

The transmembrane voltage $(\Delta \phi)$ is therefore a critical parameter for cell permeabilization [10]. The absolute value of the threshold transmembrane voltage $\left(\Delta \phi_{\mathrm{c}}\right)$ is in the range of $200-1000 \mathrm{mV}[8,11-14]$. Since $\Delta \phi_{\mathrm{c}}$ can in many cases be considered much larger than the resting transmembrane voltage $\left(\Delta \phi_{\mathrm{r}}\right)$ (which is between -20 and $-200 \mathrm{mV}$ [15]), in most cases $\Delta \phi_{\mathrm{r}}$ is not taken into account. To verify if such a simplification is justified, we evaluated the importance of $\Delta \phi_{\mathrm{r}}$ on the cell permeabilization. As a parameter that determines the extent of permeabilization, we chose the area of the cell membrane having $|\Delta \phi|>\Delta \phi_{\mathrm{c}}$ since permeabilization occurs only on the part of the cell membrane where $\Delta \phi$ exceeds the threshold value, i.e. the area which represents the pathway for transport of molecules through the membrane [16-19].

The asymmetry of permeabilization due to $\Delta \phi_{\mathrm{r}}$ was observed in several experiments $[7,8,20-23]$ where in some cases only permeabilization on the anodic side of the cell was observed. We therefore analyzed also the difference in permeabilized area on the anodic and cathodic side of the cell membrane and evaluated this effect for different parameters. 


\section{Materials and methods}

The induced transmembrane voltage $\left(\Delta \phi_{\mathrm{i}}\right)$ was calculated numerically for a sphere and for prolate and oblate spheroids having different ratios of radii $\rho=R_{1} / R_{2}$ at various angles of cell orientation $\alpha$ with respect to the applied electric field (Fig. 1). For cells that slightly differ from spherical shape ratio $\rho=10 / 8$ was used for prolate spheroid ( $\rho=8 / 10$ for oblate spheroid). In the case of cells that deviate more from spherical shape, the ratios $\rho=10 / 5(5 / 10)$ and 10/2 (2/10) were used (like plated $\mathrm{CHO}$ cells, which are approximately $40 \mu \mathrm{m}$ long and $20 \mu \mathrm{m}$ wide; bacillus, which are $4 \mu \mathrm{m}$ long and $0.8 \mu \mathrm{m}$ wide; and erythrocytes, which are similar to $2 \mu \mathrm{m}$ long and $8 \mu \mathrm{m}$ wide oblate spheroid).

All calculations were performed for only one size of the cells, however, due to the linearity of the problem, the results for all other cell sizes can be obtained by rescaling the results obtained for our specific case. If we have a cell, which is two times larger, then the same $\Delta \phi_{\mathrm{i}}$ and consequentially the percentage of the area of the cell membrane having $|\Delta \phi|>\Delta \phi_{\mathrm{c}}$ will be achieved at two times lower electric field strength, since $\Delta \phi$ is proportional to $E \times R$, where $R$ is the larger of both radii. In all our models we set $R$ to be $10 \mu \mathrm{m}$ (for prolate spheroid $R=R_{1}$, for oblate spheroid $R=R_{2}$ ).

A cell was modeled as a non-conductive spheroid placed in a conductive medium. By applying positive and negative potential of the same absolute value on the two opposite sides of the box we generated homogenous electric field. More detailed description of the model is given in Valič et al. [24].

If no $\Delta \phi_{\mathrm{r}}$ is included in the model, the centre of the cell has potential zero volts due to the symmetry of the geometry. $\Delta \phi_{\mathrm{r}}$ was superimposed by setting the centre of the cell on the preferred value of $\Delta \phi_{\mathrm{r}}, 0$ or $-100 \mathrm{mV}$.

The object of the analysis was the area of the cell membrane which is permeabilized, i.e. where membrane permeability is increased due to the exposure to the applied electric field. Therefore, we calculated $\Delta \phi$ for the above mentioned cell geometries and calculated the

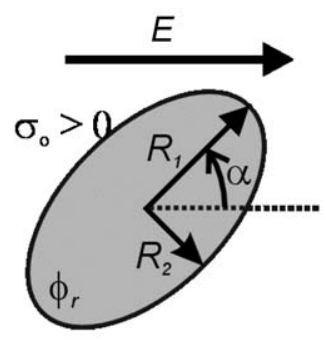

a

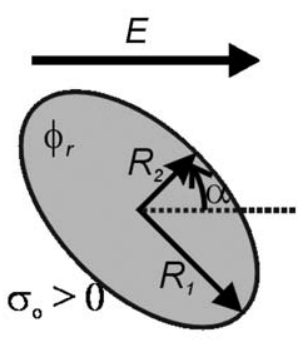

b
Fig. 1. Cross-section of a prolate (a) and oblate (b) spheroid exposed to the applied electric field. $\alpha$ is the angle of cell orientation between the applied electric field $E$ and the axis of symmetry, which is in the case of a prolate spheroid parallel to $R_{1}$ and in the case of oblate spheroid parallel to $R_{2}$. area of the cell membrane having $|\Delta \phi|>\Delta \phi_{\mathrm{c}}$ for different angles of cell orientation $\alpha\left(0^{\circ}, 15^{\circ}, 30^{\circ}, 45^{\circ}, 60^{\circ}, 75^{\circ}\right.$ and $90^{\circ}$ ). We used a static model, where $\Delta \phi$ is calculated at the moment after the transient and before the permeabilization takes place therefore membrane is assumed to be non-conductive.

A finite-elements model of a cell in a conducting medium was built using a program package of Ansoft (Pittsburgh, PA, USA), Maxwell. Additional drawings and comparison between results were performed with Matlab (The MathWorks, Massachusetts, USA).

\section{Results}

The induced transmembrane voltage $\left(\Delta \phi_{\mathrm{i}}\right)$ was calculated numerically for different cell orientations and applied electric field strengths from 100 to $700 \mathrm{~V} / \mathrm{cm}$ in steps of $100 \mathrm{~V} / \mathrm{cm}$. By superimposing $\Delta \phi_{\mathrm{i}}$ to the resting transmembrane voltage $\left(\Delta \phi_{\mathrm{r}}\right)$ we obtained transmembrane voltage $(\Delta \phi)$. As the area which will be permeabilized we defined the area of the cell membrane having $|\Delta \phi|>\Delta \phi_{\mathrm{c}}$ for different $\Delta \phi_{\mathrm{c}}=200,300,400,500 \mathrm{mV}$.

In Fig. 2, the percentage of the area of the cell membrane having $|\Delta \phi|>\Delta \phi_{\mathrm{c}}$ for a sphere and for prolate spheroids having ratios $\rho=10 / 5$ and $10 / 2$ is presented for different angles of cell orientation $\alpha$. On the left side (a, c, e), data for applied electric field $E=100,200$ and $300 \mathrm{~V} / \mathrm{cm}$ are shown for $\Delta \phi_{\mathrm{c}}=200 \mathrm{mV}$, whereas on the right side (b, d, f) data for applied electric field $E=300,500$ and $700 \mathrm{~V} / \mathrm{cm}$ are shown for $\Delta \phi_{\mathrm{c}}=500 \mathrm{mV}$. For each applied electric field strength two cases were examined. One, when $\Delta \phi_{\mathrm{r}}$ is zero, and the other, when $\Delta \phi_{\mathrm{r}}$ is $-100 \mathrm{mV}$ (denoted with index r). In Fig. 2, a and $b$ data for a sphere are presented. As expected the angle of cell orientation $\alpha$ due to the spherical symmetry has no effect on the area of the cell membrane having $|\Delta \phi|>\Delta \phi_{\mathrm{c}}$. Altogether, the value of $\Delta \phi_{\mathrm{r}}$ is not important when $\Delta \phi_{\mathrm{i}} \gg \Delta \phi_{\mathrm{c}}$, However, when $\Delta \phi_{\mathrm{i}}$ is close to $\Delta \phi_{\mathrm{c}}$ we can see that $\Delta \phi_{\mathrm{r}}$ reduces the applied electric field strength needed for permeabilization. For an ideal non-conductive membrane and steady-state conditions, the transmembrane voltage is described by:

$\Delta \phi_{i}=f E R \cos \vartheta$.

From the above equation, $\Delta \phi_{\mathrm{i}}$ is calculated for the applied electric field and added to $\Delta \phi_{\mathrm{r}}$. Therefore the $\max \left|\Delta \phi_{\text {anodic }}\right|=\max \left|\Delta \phi_{\mathrm{i}}\right| \pm\left|\Delta \phi_{\mathrm{r}}\right|$ will give for a sphere $(f=1.5)$ in the caase of $\Delta \phi_{\mathrm{r}}=100 \mathrm{mV} 550 \mathrm{mV}$ on anodic side and 350 $\mathrm{mV}$ on cathodic side. Therefore if $\Delta \phi_{\mathrm{c}}=500 \mathrm{mV}$ a part of the cell membrane is permeabilized, whereas in the case of $\Delta \phi_{\mathrm{r}}=0 \mathrm{mV} \max \Delta \phi_{\text {anodic }}=\Delta \phi_{\text {cathodic }}=450 \mathrm{mV}$ and no permeabilization occurs. For comparison with $\Delta \phi_{\mathrm{c}}$ the corresponding $\Delta \phi_{\mathrm{i}}$ for the applied electric field is presented in legend of Fig. 2, calculated from the depolarizing factors given in Valič et al. [24]. 


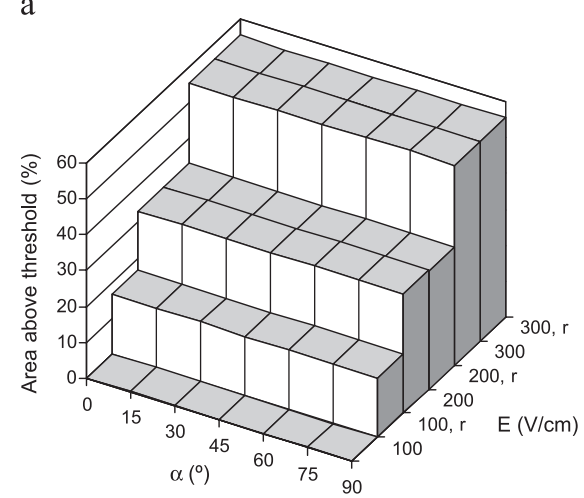

c

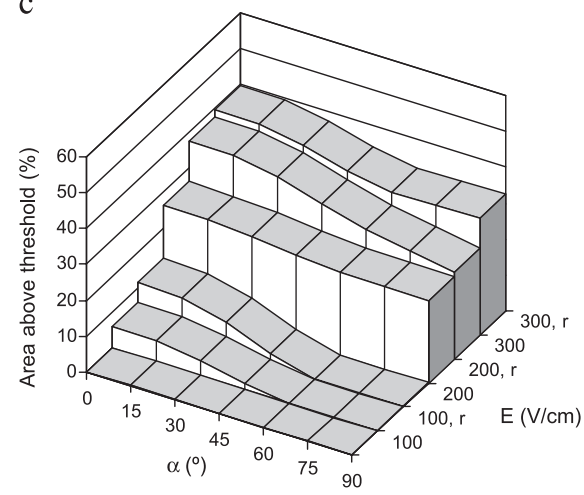

e

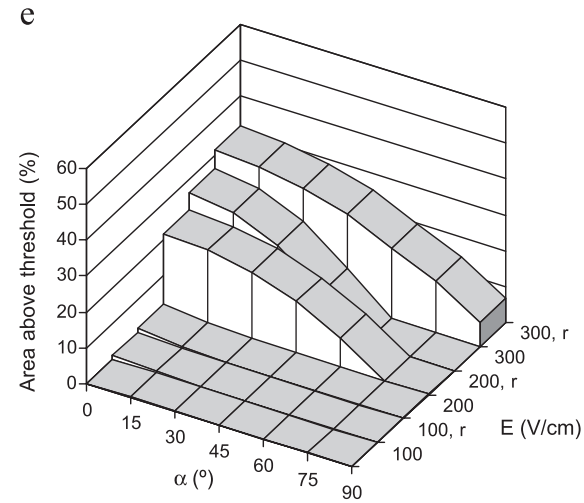

b

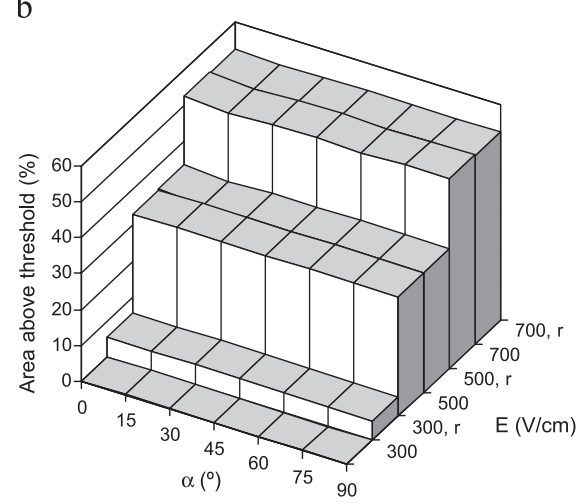

d

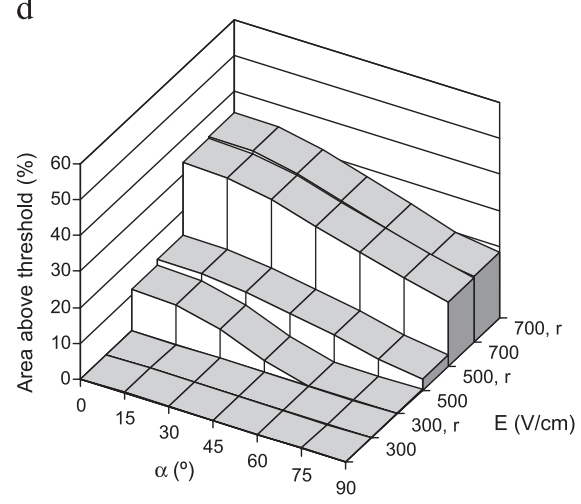

$\mathrm{f}$

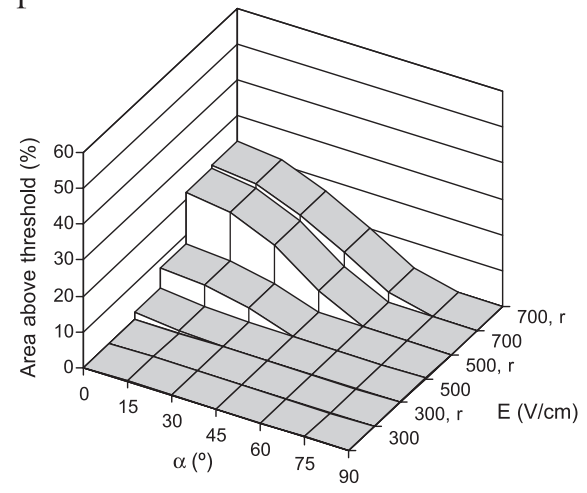

Fig. 2. The percentage of the area of the cell membrane having the absolute value of the transmembrane voltage higher than the threshold value of the transmembrane voltage $\left(\Delta \phi>\Delta \phi_{\mathrm{c}}\right)$ for a sphere (a and b), for prolate spheroid with ratio $\rho=10 / 5$ (c and d) and for prolate spheroid with ratio $\rho=10 / 2$ (e and f) is presented respectively for different cell orientation $\alpha$. The non-zero resting transmembrane voltage $\left(\Delta \phi_{\mathrm{r}}=-100 \mathrm{mV}\right)$ is denoted by $\mathrm{r}$, else $\Delta \phi_{\mathrm{r}}=-0 \mathrm{mV}$. On the left side (a, c, e) $\Delta \phi_{\mathrm{c}}$ is $200 \mathrm{mV}$ and on the right side (b, d, f) $500 \mathrm{mV}$. Applied electric field strength [100, 200, 300, 500, 700] V/cm corresponds to maximum induced transmembrane potential $\max \Delta \phi_{\mathrm{i}}=[150,300,450,750,1050] \mathrm{mV}$ on a sphere, to $[120,240,360,600,840] \mathrm{mV}$ on a prolate spheroid with ratio $\rho=10 / 5$ and to $[120,240,360,600,840] \mathrm{mV}$ on a prolate spheroid with ratio $\rho=10 / 5$.

By comparing Fig. 2a to $2 \mathrm{~b}$, we can see that $\Delta \phi_{\mathrm{r}}$ is more important in the cases where $|\Delta \phi|$ is only a little higher than $\Delta \phi_{\mathrm{c}}$ and when $\Delta \phi_{\mathrm{r}}$ is close to the $\Delta \phi_{\mathrm{c}}$.

Results for spheroidal cell (shown in Fig. 2c,d,e,f) for $\rho=10 / 5$ and $10 / 2$ are similar to the results for the sphere with some differences: (i) lower average area of the cell membrane having $|\Delta \phi|>\Delta \phi_{\mathrm{c}}$, since $\Delta \phi_{\mathrm{i}}$ depends also on geometric factor $f$; (ii) for spheroidal cells the orientation angle $\alpha$ significantly influences the area of the cell membrane having $|\Delta \phi|>\Delta \phi_{\mathrm{c}}$. This effect is more pronounced for cells that deviate more from spherical shape.

Another important effect of the $\Delta \phi_{\mathrm{r}}$ is the difference between the permeabilized area of the cathodic and anodic side. When $\Delta \phi_{\mathrm{r}}$ is zero, both areas are equal. However, if $\Delta \phi_{\mathrm{r}}=-100 \mathrm{mV}$, then the area of the cell membrane having $|\Delta \phi|>\Delta \phi_{\mathrm{c}}$ on the anodic side is larger than the permeabilized area on the cathodic side (Fig. 3). This is 

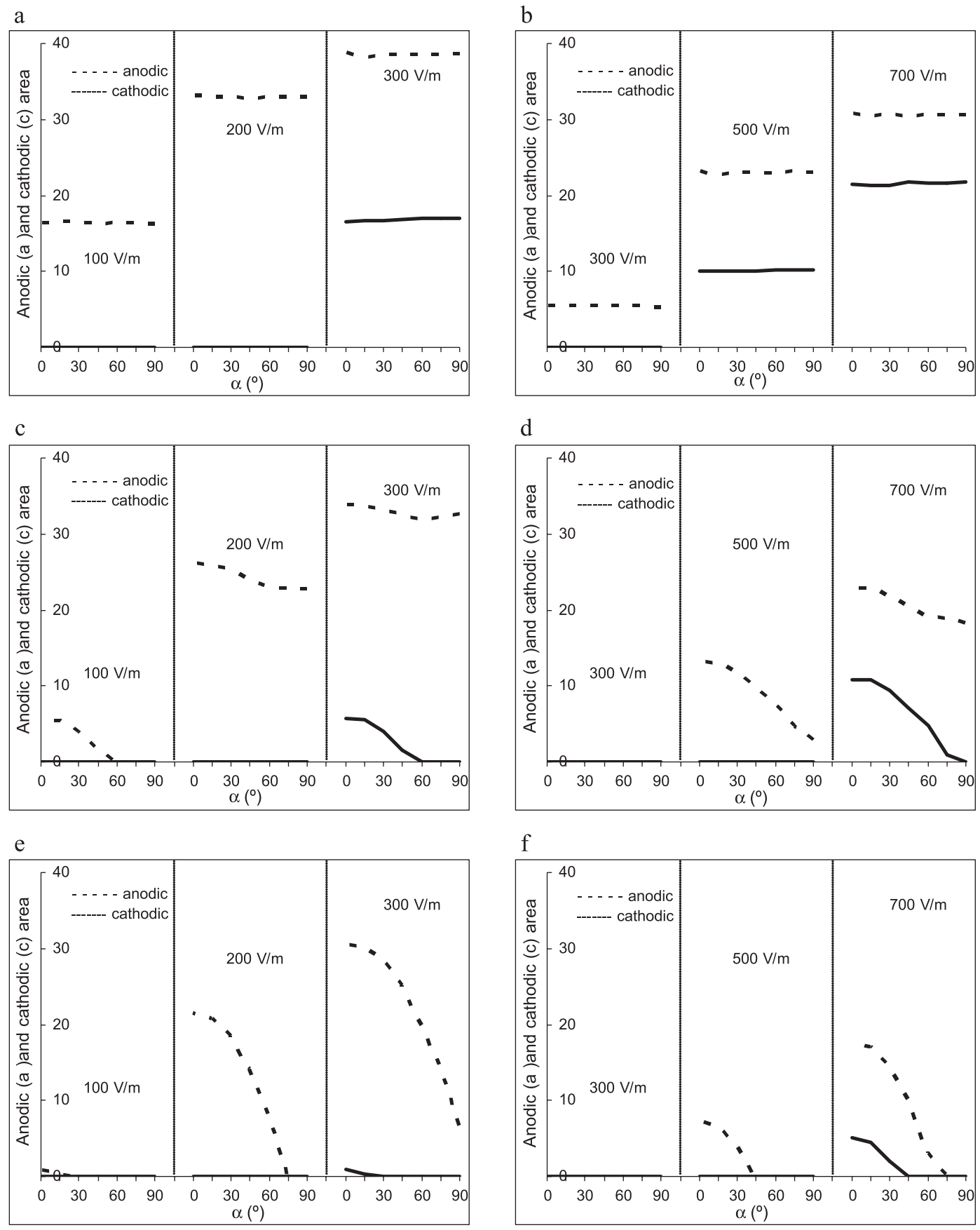

Fig. 3. Comparison of anodic (solid line) and cathodic (dashed line) area of the cell membrane having the absolute value of the transmembrane voltage higher than the threshold value of the transmembrane voltage $\left(\Delta \phi>\Delta \phi_{\mathrm{c}}\right)$ for resting transmembrane voltage $\Delta \phi_{\mathrm{r}}=-100 \mathrm{mV}$. Data for a sphere (a and b), for prolate spheroid with $\rho=10 / 5$ (c and d) and 10/2 (e and f) is presented for different angles of cell orientation $\alpha$. On the left side (a, c, e) $\Delta \phi_{\mathrm{c}}$ is $200 \mathrm{mV}$ and on the right side $(\mathrm{b}, \mathrm{d}, \mathrm{f}) \Delta \phi_{\mathrm{c}}$ is $500 \mathrm{mV}$.

due to the fact that $\Delta \phi_{\mathrm{r}}$ is negative and when it is superimposed to the $\Delta \phi_{\mathrm{i}}$, which is also negative on the part of the cell membrane facing the anode, there is higher $|\Delta \phi|$ on the anodic side than on the cathodic side. Thus, permeabilization occurs first at the anodic side, as was demonstrated experimentally in a number of experiments $[7,8,16,20-22]$.

\section{Discussion and conclusion}

The area of the cell membrane where the transmembrane voltage $(\Delta \phi)$ exceeds the threshold value $\left(\Delta \phi_{c}\right)$ represents the pathway for transport of molecules in electropermeabilized cells [16-19]. In the presented study we theoretically analyzed the effect of cell geometry and orientation, resting 
transmembrane voltage $\left(\Delta \phi_{\mathrm{r}}\right)$ and applied electric field strength, on the area having $|\Delta \phi|>\Delta \phi_{\mathrm{c}}$. The values of $\Delta \phi_{\mathrm{r}}$ and $\Delta \phi_{\mathrm{c}}$ were varied in a range given in literature; however the data for different cells exhibit large differences. Therefore, for specific experimental conditions realistic values of the $\Delta \phi_{\mathrm{r}}, \Delta \phi_{\mathrm{c}}$ and cell shape have to be taken into account.

Based on the results which we obtained we can conclude that the major factor which determines the importance of $\Delta \phi_{\mathrm{r}}$ is the ratio between $\Delta \phi_{\mathrm{i}}$ and $\Delta \phi_{\mathrm{c}}$. If $\Delta \phi_{\mathrm{i}} \gg \Delta \phi_{\mathrm{c}}$ the $\Delta \phi_{\mathrm{r}}$ has negligible effect on the area of the cell membrane having $|\Delta \phi|>\Delta \phi_{\mathrm{c}}$, i.e. on the permeabilized area. However, in most of the experimental conditions $\Delta \phi_{\mathrm{i}}$ has to be near the $\Delta \phi_{\mathrm{c}}$ to preserve cell viability therefore in this case the influence of $\Delta \phi_{\mathrm{r}}$ is significant. In Fig. 2, it can be seen that the orientation of the spheroidal cell in applied electric field affects the area of the cell membrane having $|\Delta \phi|>\Delta \phi_{\mathrm{c}}$, the effect being larger for cells that deviate more from spherical shape as already shown in our previous paper [24]. In general, however, the effect of resting transmembrane potential is similar for different orientations.

Furthermore, we evaluated for different parameters the effect of $\Delta \phi_{\mathrm{r}}$ on the difference between the cathodic and anodic area of the cell membrane having $|\Delta \phi|>\Delta \phi_{\mathrm{c}}$. Again, the asymmetry is more pronounced when $\Delta \phi_{\mathrm{i}}$ is close to $\Delta \phi_{\mathrm{c}}$, however, the asymmetry is not significantly affected by the angle of cell orientation $\alpha$.

The calculated asymmetry of the area of the cell membrane having $|\Delta \phi|>\Delta \phi_{\mathrm{c}}$ agrees with the observed asymmetric $\Delta \phi$ distribution and uptake of molecules [7,8,16,20-23].

It was already suggested previously by Kotnik et al. [25] that by using bipolar pulses instead of unipolar pulses, the area of the cell membrane having $|\Delta \phi|>\Delta \phi_{\mathrm{c}}$ and by this also electropermeabilization can be increased for the same applied electric field while preserving the viability of cells. Our calculations support this suggestion that for $\Delta \phi_{\mathrm{i}}$ in the range of $\Delta \phi_{\mathrm{c}}$ or relatively high $\Delta \phi_{\mathrm{r}}$ compared to $\Delta \phi_{\mathrm{c}}$, bipolar pulses can significantly increase the efficiency of permeabilization.

\section{Acknowledgements}

This research was in part supported by the Ministry of Education, Science and Sports of the Republic of Slovenia, and the European Commission, under the grant Cliniporator QLK3-99-00484 within the 5th framework.

\section{References}

[1] E. Neumann, K. Rosenheck, Permeability changes induced by electric impulses in vesicular membranes, J. Membr. Biol. 10 (1972) 279-290.

[2] U. Zimmermann, Electric field-mediated fusion and related electrical phenomena, Biochim. Biophys. Acta 694 (1982) 227-277.

[3] E. Neumann, A.E. Sowers, C.A. Jordan, Electroporation and Electrofusion in Cell Biology, Plenum, New York, 1989.
[4] T.Y. Tsong, Electroporation of cell membranes, Biophys. J. 60 (1991) 297-306.

[5] J.C. Weaver, Y.A. Chizmadzhev, Theory of electroporation, Bioelectrochem. Bioenerg. 41 (1996) 135-160.

[6] L.M. Mir, Therapeutic perspectives of in vivo cell electropermeabilization, Bioelectrochemistry 53 (2001) 1-10.

[7] E. Tekle, R.D. Astumian, P.B. Chock, Electro-permeabilization of cell membranes: effect of the resting membrane potential, Biochem. Biophys. Res. Commun. 172 (1990) 282-287.

[8] J. Teissié, M.P. Rols, An experimental evaluation of the critical potential difference inducing cell membrane electropermeabilization, Biophys. J. 65 (1993) 409-413.

[9] E. Neumann, S. Kakorin, K. Toensig, Fundamentals of electroporative delivery of drugs and genes, Bioelectrochem. Bioenerg. 48 (1999) 3-16.

[10] M.P. Rols, J. Teissié, Electropermeabilization of mammalian cells to macromolecules: control by pulse duration, Biophys. J. 75 (1998) $1415-1423$.

[11] J.C. Weaver, K.T. Powell, Theory of electroporation, in: E. Neumann, A.E. Sowers, C.A. Jordan (Eds.), Electroporation and Electrofusion in Cell Biology, Plenum, New York, 1989, pp. 111-112.

[12] N. Eynard, F. Rodriguez, J. Trotard, J. Teissié, Electrooptics studies of Escherichia coli electropulsation: orientation, permeabilization, and gene transfer, Biophys. J. 75 (1998) 2587-2596.

[13] M. Bier, S.M. Hammer, D.J. Canaday, R.C. Lee, Kinetics of sealing for transient electropores in isolated mammalian skeletal muscle cells, Bioelectromagnetics 20 (1999) 194-201.

[14] D. Miklavčič, D. Šemrov, H. Mekid, L.M. Mir, A validated model of in vivo electric field distribution in tissues for electrochemotherapy and for DNA electrotransfer for gene therapy, Biochim. Biophys. Acta 1523 (2000) 73-83.

[15] B. Alberts, D. Bray, J. Lewis, M. Raff, K. Roberts, J.D. Watson, Molecular Biology of the Cell, Garland Publishing, New York, USA, 1994

[16] M. Hibino, M. Shigemori, H. Itoh, K. Nagayama, K. Kinosita, Membrane conductance of an electroporated cell analyzed by submicrosecond imaging of transmembrane potential, Biophys. J. 59 (1991) 209-220.

[17] B. Gabriel, J. Teissié, Fluorescence imaging in the millisecond time range of membrane electropermeabilisation of single cells using a rapid ultra-low-light intensifying detection system, Eur. Biophys. J. 27 (1998) 291-298.

[18] J. Teissié, N. Eynard, B. Gabriel, M.P. Rols, Electropermeabilization of cell membranes, Adv. Drug Deliv. Rev. 35 (1999) 3-19.

[19] M. Puc, T. Kotnik, L.M. Mir, D. Miklavčič, Quantitative model of small molecules uptake after in vitro cell electropermeabilization, Bioelectrochemistry 60 (2003) $1-10$.

[20] W. Mehrle, U. Zimmermann, R. Hampp, Evidence for asymmetrical uptake of fluorescent dyes through electro-permeabilized membranes of avena mesophyll protoplasts, FEBS 185 (1985) 89-94.

[21] M. Hibino, H. Itoh, K. Kinosita, Time courses of cell electroporation as revealed by submicrosecond imaging of transmembrane potential, Biophys. J. 64 (1993) 1789-1800.

[22] B. Gabriel, J. Teissié, Direct observation in the millisecond time range of fluorescent molecule asymmetrical interaction with the electropermeabilized cell membrane, Biophys. J. 73 (1997) 2630-2637.

[23] M.N. Teruel, T. Meyer, Electroporation-induced formation of individual calcium entry sites in the cell body and processes of adherent cells, Biophys. J. 73 (1997) 785-796.

[24] B. Valič, M. Golzio, M. Pavlin, A. Schatz, C. Faurie, B. Gabriel, J. Teissié, M.P. Rols, D. Miklavčič, Electric field induced transmembrane potential on spheroidal cell: theory and experiment, Eur. Biophys. J. 32 (2003) 519-528.

[25] T. Kotnik, L.M. Mir, K. Flisar, M. Puc, D. Miklavčič, Cell membrane electropermeabilization by symmetrical bipolar rectangular pulses: Part I. Increased efficiency of permeabilization, Bioelectrochemistry 54 (2001) 83-90. 